\title{
Head-to-head comparison of in-house produced CD19 CAR-T cell in ALL and NHL patients
}

Orit Itzhaki, ${ }_{1}^{1}$ Elad Jacoby, ${ }^{2,3}$ Abraham Nissani, ${ }^{1}$ Michal Levi, ${ }^{1}$ Arnon Nagler, ${ }^{4}$ Adva Kubi, ${ }_{1}^{1}$ Karin Brezinger, ${ }^{1}$ Hadar Brayer, ${ }^{1}$ Li-at Zeltzer, ${ }^{1}$ Meir Rozenbaum, ${ }^{5}$ Helly Vernitsky, ${ }^{6}$ Gal Markel, ${ }^{1,5}$ Amos Toren, ${ }^{2,3}$ Abraham Avigdor, ${ }^{4}$ Jacob Schachter, ${ }^{1}$ Michal J Besser (1) ${ }^{1,5,7}$
To cite: Itzhaki 0, Jacoby E, Nissani A, et al. Head-tohead comparison of in-house produced CD19 CAR-T cell in ALL and NHL patients. Journal for ImmunoTherapy of Cancer 2020;8:e000148. doi:10.1136/ jitc-2019-000148

Accepted 02 February 2020

Check for updates

(c) Author(s) (or their employer(s)) 2020. Re-use permitted under CC BY-NC. No commercial re-use. See rights and permissions. Published by BMJ.

${ }^{1}$ Ella Lemelbaum Institute for Immuno Oncology, Sheba Medical Center, Tel Hashomer, Israel

${ }^{2}$ Division of Pediatric

Hematology and Oncology,

The Edmond and Lily Safra Children's Hospital, Sheba Medical Center, Tel Hashomer, Israel

${ }^{3}$ Sackler School of Medicine, Tel Aviv University, Tel Aviv, Israel ${ }^{4}$ Department of Bone Marrow Transplantation, Sheba Medical Center, Tel Hashomer, Israel ${ }^{5}$ Department of Clinical

Microbiology and Immunology, Sackler School of Medicine, Te Aviv University, Tel Aviv, Israel

${ }^{6}$ Hematology Laboratory, Sheba Medical Center, Tel Hashomer, Israel

${ }^{7}$ Wohl Institute for Translational Medicine, Sheba Medical Center, Tel Hashomer, Israel

\section{Correspondence to} Dr Michal J Besser;

Michal.Besser@sheba.health. gov.il

\section{ABSTRACT}

Background CD19 chimeric antigen receptor T (CAR-T) cells demonstrate remarkable remission rates in pediatric and adult patients with refractory or relapsed $(r / r)$ acute lymphoblastic leukemia (ALL) and non-Hodgkin's Iymphoma (NHL). In 2016, we initiated a clinical trial with in-house produced CD19 CAR-T cells with a CD28 co-stimulatory domain. We analyzed, for the first time, differences in production features and phenotype between ALL and NHL patients.

Methods Non-cryopreserved CAR-T cells were produced from patients' peripheral blood mononuclear cells within 9 to 10 days. 93 patients with $\mathrm{r} / \mathrm{r} \mathrm{ALL}$ and NHL were enrolled under the same study. CAR-T cells of ALL and NHL patients were produced simultaneously, allowing the head-to-head comparison.

Results All patients were heavily pretreated. Three patients dropped out from the study due to clinical deterioration $(n=2)$ or production failure $(n=1)$. Cells of ALL patients $(n=37)$ expanded significantly better and contained more CAR-T cells than of NHL patients $(n=53)$. Young age had a positive impact on the proliferation capacity. The infusion products from ALL patients contained significantly more naïve CAR-T cells and a significantly higher expression of the chemokine receptor CXCR3. PD-1, LAG-3, TIM-3, and CD28 were equally expressed. $100 \%$ of ALL patients and $94 \%$ of NHL patients received the target dose of $1 \times 10 \mathrm{e} 6 \mathrm{CAR}-\mathrm{T} / \mathrm{kg}$. The overall response rate was $84 \%(30 / 36)$ in $A L L$ and $62 \%(32 / 52)$ in NHL. We further compared CAR-T cell infusion products to tumor infiltrating lymphocytes (TIL), another common type of T cell therapy, mainly clinically effective in solid tumors. CAR-T cells contained significantly more naïve T cells and central memory $T$ cells and significantly less CCR5 compared to TIL infusion products.

Conclusions The in-house production of CAR-T cells is highly efficient and fast. Clinical response rate is high. CAR-T cells can be successfully produced for $99 \%$ of patients in just 9 to 10 days. Cells derived from ALL patients demonstrate a higher proliferation rate and contain higher frequencies of CAR-T cells and naïve T cells than of NHL patients. In addition, understanding the differences between CAR-T and TIL infusion products, may provide an angle to develop CAR-T cells for the treatment of solid tumors in the future.
Trial registration number ClinicalTrials.gov; CAR-T: NCT02772198, First posted: May 13, 2016; TIL: NCT00287131, First posted: February 6, 2006.

\section{INTRODUCTION}

Adoptive cell therapy (ACT) with autologous CD19 chimeric antigen receptor $\mathrm{T}$ cells (CAR-T) has demonstrated remarkable remission rates in patients with relapsed and refractory $(\mathrm{r} / \mathrm{r})$ B-cell malignancies. The CAR combines a single chain variable fragment ectodomain of an antibody ( $\mathrm{scFv}$ ) that can target an antigen of choice with an endodomain $\mathrm{T}$ cell signaling moiety comprised of the CD3 $\zeta$ TCR signal and additional co-stimulatory domains. In contrast to the $\mathrm{T}$ cell receptor, CAR induces antigen recognition in a major histocompatibility complex (MHC)-independent manner. ${ }^{1}$ The CAR-T approach was pioneered in the late $1980 \mathrm{~s}^{23}$ and finally let to its approval by the Food and Drug Administration (FDA) in 2017 and the European Medicines Agency (EMA) in 2018.

Currently two commercial CD19 CAR-T products exist on the market for the treatment of B-cell precursor acute lymphoblastic leukemia (ALL) and various types of nonHodgkin's lymphoma (NHL). Both CAR-T products, tisagenlecleucel and axicabtagene ciloleucel, were approved for adult patients with $\mathrm{r} / \mathrm{r}$ B-cell lymphoma including diffuse large B-cell lymphoma (DLBCL), high grade B-cell lymphoma and DLBCL arising from follicular lymphoma. ${ }^{4}$ In addition, tisagenlecleucel was approved for patients up to 25 years of age with $\mathrm{r} / \mathrm{r}$ ALL. ${ }^{5-7}$

ALL is the most common cancer in childhood and young adults. In children the 5 year survival rate approaches $90 \%$ with standard treatment, but clinical results for relapsed disease remains poor. ${ }^{8}$ The majority of adults 
with ALL who achieve complete response (CR) with standard therapies will relapse after first remission and about $25 \%$ will have refractory disease, leading to high mortality. ${ }^{9}$

Non-Hodgkin's lymphomas are a heterogeneous group of malignancies with distinct prognoses and therapeutic approaches. NHL is the most frequent hematological malignancies in the world with more than 50,000 new cases in USA per year. ${ }^{10}$ It consists of 40 major subtypes with distinct genetic, morphologic and clinical features. ${ }^{11}$ DLBCL is the most common subtype, holding $25 \%$ to $30 \%$ of adult NHL in Western countries, ${ }^{12}$ however many additional and rare CD19 positive lymphoma types, such as Burkitt lymphoma exist, which do not fall under the approved indications of CD19 CAR-T cells. The same is true for chronic lymphocytic leukemia, the most common type of leukemia in adults, accounting for $30 \%$ of cases. ${ }^{13}$

The addition of CD19 CAR-T cells to the existing treatment repertoire of $r / r$ ALL and various types of NHL was a major breakthrough, however there are still obstacles related to the commercial CAR-T cell products, including high costs and a long turnaround time from leukapheresis to infusion of 21 to 60 days.

In-house production of CAR-T cells can overcome most of these obstacles, leading to a raising number of clinical centers generating their own CAR-T products today. In 2016, the Sheba Medical Center, Israel, initiated a phase 1b/2 study with in-house produced CD19 CAR-T cells for the treatment of CD19 positive B-cell malignancies. Patients undergo a single leukapheresis procedure. Peripheral blood mononuclear cells (PBMC) are isolated, activated and transduced with a gamma retrovirus encoding for the anti CD19 scFv, a CD28 co-stimulatory domain and a CD3- $\zeta$ signaling domain. Following expansion, fresh, non-cryopreserved CAR-T cells are administered to the patients after a turnaround time of only 9 to 10 days. In contrast to the commercial products, also patients with off-label CD19 positive B-cell malignancies, such as adults with $\mathrm{r} / \mathrm{r}$ ALL and Burkitt lymphoma were enrolled to the study and CAR-T cells were manufactured in just 10 days. Clinical results of the first 21 patients with $\mathrm{r} / \mathrm{r}$ ALL and one patient with Burkitt lymphoma were previously published. ${ }^{1415}$

Here, we describe the manufacturing process of 91 in-house produced CAR-T products. Patients with $\mathrm{r} / \mathrm{r}$ ALL and NHL were enrolled under the same study and CAR-T were produced in parallel, allowing true head-tohead comparison of CAR-T cell production and characterization in ALL and NHL.

\section{MATERIAL AND METHODS Patients}

This study was designed as a phase $1 \mathrm{~b} / 2$ trial, approved by the Israeli Ministry of Health and registered at ClinicalTrials.gov. All enrolled patients signed an informed consent. Inclusion criteria were age between 1 and 50 years, failure of at least two prior therapeutic protocols, a CD3 count greater than $250 / \mu \mathrm{l}$ blood, absence of clinical signs of graft-versus-host disease and no immunosuppressive treatment. Patients above age 50 were permitted to the study after approval by the Sheba Medical Center Institutional Review Board (IRB). Depending on age, the minimal performance score was 50 on a Lansky scale or on a Karnofsky scale. Patients with prior CD19 directed therapies were eligible for the study. Lympho-depleting conditioning was inducted by fludarabine $25 \mathrm{mg} / \mathrm{m}^{2}$ for 3 days (2 to 4 days before infusion) and cyclophosphamide $900 \mathrm{mg} / \mathrm{m}^{2}$ for 1 day (2 days before infusion), followed by infusion of $1 \times 10 \mathrm{e} 6$ transduced CAR-T cells per kilogram weight. Primary endpoints of the study were production feasibility, patient safety and best overall response rates, documented 1 to 2 months after infusion.

\section{CD19 CAR retroviral vector}

The retroviral supernatant was generated from the CD19 CAR producer line PG13-CD19-CAR-H3 kindly provided by Dr. Steven Rosenberg, National Cancer Institute (NCI). Vector construction and master cell bank production were conducted at the NCI. ${ }^{1617}$ In short, a plasmid encoding the CD19 CAR containing of the mouse stem-cell virus gamma-retroviral backbone engineered to a $\mathrm{scFv}$ derived from the mouse anti-CD19 hybridoma, FMC63, ${ }^{18}$ fused to intracellular domains from human CD28 and CD3- $\zeta$, was used for viral vector production. Retroviral vector supernatant was harvested in accordance with current good manufacturing practices at the Indiana University Viral Production Facility.

\section{CAR-T production}

If not otherwise indicated, a fresh leukapheresis product was used as starting material for CD19 CAR-T cell production. PBMC were isolated from the apheresis product by density gradient with Ficoll-Hypaque (Lymphocyte Separation Medium, Axis-Shield Diagnostics, Scotland). 400×10e6 PBMC were re-suspended at the concentration of $1 \times 10 \mathrm{e} 6$ cells per $\mathrm{ml}$ in complete medium (CM), containing $10 \%$ human AB serum (Valley, Virginia, USA), $2 \mathrm{mM}$ L-Glutamine (Biological Industries, Israel), Pen/Strep (Biological Industries, Israel) and $300 \mathrm{IU} / \mathrm{mL}$ IL-2 (Chiron Novartis, New Jersey, USA) in AIM-V medium (Invitrogen, California, USA). $50 \mathrm{ng} / \mathrm{mL}$ anti-CD3 monoclonal antibody OKT-3 (Miltenyi Biotec, Bergisch Gladbach, Germany) were added to the medium on the day of initiation. After 2 days around $60 \times 10 \mathrm{e} 6$ cells were transduced with the CD19 CAR retroviral vector and the rest of the cells discarded. For this purpose, non-tissue culture treated 6-well plates were coated with $10 \mu \mathrm{g} / \mathrm{mL}$ RetroNectin (Takara Bio Inc, Otsu, Japan) in phosphote-buffered saline (PBS) for 2 hours at room temperature or overnight at $4^{\circ} \mathrm{C}$, followed by $30 \mathrm{~min}$ blocking with 2.5\% human albumin (Bio Products Laboratory, Zenalb20) in PBS and washed. Retroviral supernatant was thawed and diluted 1:1 in AIM-V medium with 5\% human AB serum. Four $\mathrm{ml}$ of the diluted vector were added per well of the retronectin-coated plates and centrifuged at 2000xg for 2 hours at $32^{\circ} \mathrm{C}$. The supernatant was aspirated 
and cells were re-suspended in CM medium with IL-2. $2.5 \times 10 \mathrm{e} 6$ cells were added to each well (total of $60 \times 10 \mathrm{e} 6$ cells in four 6 -well plates), centrifuged for $15 \mathrm{~min}$ at $1000 \mathrm{xg}$ and incubated at $37^{\circ} \mathrm{C}$ overnight. On day 3 the cells were transferred to T175 or GRex100 (Wilson Wolf, Minnesota, USA $)^{19}$ culture flasks and maintained at a concentration of 0.5 to $2.0 \times 10 \mathrm{e} 6$ cells $/ \mathrm{mL}$ in T175 flasks or 0.5 to $8.0 \times 10 \mathrm{e} 6$ cells $/ \mathrm{mL}$ in GRex100 in CM medium with IL-2 until day 9 or 10. When using GRex100, flasks were topped up to 450 $\mathrm{ml}$ with IL-2 containing $\mathrm{CM}$ medium on day 6 . On the day of infusion, cells were washed, counted and $1 \times 10 \mathrm{e} 6 \mathrm{CD} 19$ CAR expressing cells $/ \mathrm{kg}$ were re-suspended in $100 \mathrm{~mL}$ $0.9 \%$ sodium chloride (Baxter,) containing $2.5 \%$ human albumin and $300 \mathrm{IU} / \mathrm{mL}$ IL-2. The cell product was delivered to the patient for immediate infusion. CAR-T cells for ALL and NHL patients were produced in parallel and by the same laboratory staff.

\section{TIL production}

Tumor infiltrating lymphocytes (TIL) were isolated from tumor biopsies of metastatic melanoma patients, enrolled to a phase II TIL ACT trial at the Sheba Medical Center. The generation of TIL was conducted precisely as described before. ${ }^{20}{ }^{21}$ In short, fragmentation, enzymatic digestion and tissue remnant culture techniques were used to isolate TIL from surgically resected metastatic melanoma lesions. Cells were cultured in CM medium with IL-2 $(3000 \mathrm{IU} / \mathrm{mL})$ and gave rise to TIL cultures within 2 to 4 weeks. Next, TIL were expanded in a rapid expansion procedure (REP) using anti-CD3 monoclonal antibody OKT-3 $(30 \mathrm{ng} / \mathrm{mL})$, IL-2 $(3000 \mathrm{IU} / \mathrm{mL})$ and $50 \mathrm{~Gy}$ irradiated feeder cells of healthy donors. Within 2 weeks, cultures expanded by about 1000 -fold. On day 14 , TIL were harvested, washed, re-suspended in $400 \mathrm{~mL}$ $0.9 \%$ sodium chloride containing $2.5 \%$ human albumin and $300 \mathrm{IU} / \mathrm{mL}$ IL-2 and immediately intravenously administered to the patient.

\section{In vitro reactivity}

In order to demonstrate in vitro anti-tumor reactivity, IFN $\gamma$ secretion was measured following co-incubation of CAR-T cells with target cells. ${ }^{172-25}$ Untransduced T cells served as negative control. The following CD19-expressing target cell lines were used: NALM-6 (acute lymphoid leukemia); Toledo (B-cell diffuse large cell lymphoma). The CD19negative cell line CCRF-CEM (T cell leukemia) was used as negative control. All tumor lines were kindly provided by Dr. Steven Rosenberg, NCI.

The co-culture was performed with an effector to target ratio of $1: 1\left(1 \times 10^{5}\right.$ each) in a total of $200 \mu 1$ medium overnight at $37^{\circ} \mathrm{C}$. Supernatant was collected, if necessary diluted and IFN $\gamma$ secretion was determined by ELISA (Human IFN- ELISA MAX Deluxe Set, BioLegend, San Diego, California). Measurements were performed in triplicates.

\section{Flow cytometry}

The following antibodies were used: CD3 (VioBlue; Miltenyi Biotech or Pacific blue and PE; BioLegend),
CD4 (FITC or APC-Cy7; BioLegend), CD8 (PE-Cy7; BioLegend), CD3/CD19 antibodies (FITC/PE; BD), CD28 (PerCP-Cy5.5; eBioscience), PD-1 (FITC; clone: EH12.2H7; BioLegend), TIM-3 (APC-Cy7; BioLegend), LAG-3 (VioBlue; Miltenyi Biotech), CD45RA (APC-Vio770; Miltenyi Biotec or Brilliant Violet; BioLegend), CCR7 (PerCP-Vio770; Miltenyi Biotec or PerCP; BioLegend), CCR2 (APC; Biolegend), CCR4 (PE; Biolegend), CCR5 (Alexa Influenza 488; Biolegend), CXCR2 (PE-Cy7; Biolegend) and CXCR3 (FITC; Biolegend).

Transduction efficacy was determined on day 6 and day 9 of culture by labeling CAR-T cells with biotin-labeled polyclonal goat anti-mouse $\mathrm{F}(\mathrm{ab})_{2}$ antibody (anti-Fab, Jackson Immunoresearch, West Grove, Pennsylvania) and streptavidin (APC conjugated; BioLegend). $\mathrm{CD} 3+\mathrm{F}(\mathrm{ab})_{2}+$ cells were defined as CAR-T cells. Isotype labeled cells (Jackson Immunoresearch) and untransduced cells served as negative controls. For further characterization, cells were stained with antibodies mentioned above. Cells were washed and re-suspended in cell staining buffer (BioLegend), incubated for $30 \mathrm{~min}$ with the antibodies on ice, washed and measured using MACSQuant FACS cytometer (Miltenyi Biotec). Samples were analyzed by FlowJo software (FlowJo LLC, Ashland, Oregon).

\section{Statistics}

Significance of variation between groups was evaluated using a non-parametric two-tailed Student's t-test. The differences between proportions were tested using two-sided Fisher's exact test. Analysis of covariance (ANCOVA) was used to exam the differences in the mean values of patients' characteristics on variable production parameters.

\section{RESULTS}

\section{Patients' characteristics and clinical response}

Between June 2016 and August 2019, 93 patients with $\mathrm{r} / \mathrm{r}$ B-cell malignancies were enrolled to the trial. All patients were heavily pretreated. Three enrolled patients $(3 \%)$ dropped out from the study due to clinical deterioration $(n=2)$ or failure to produce CAR-T cells $(n=1$; absence of CAR-T cells in the infusion product). One patient was treated twice. Of the treated patients, 37 patients had $\mathrm{r} / \mathrm{r}$ ALL and 53 patient's $r / r$ NHL, including DLBCL $(n=36)$, Burkitt lymphoma $(\mathrm{n}=3)$, PMBCL $(\mathrm{n}=7)$, follicular lymphoma $(n=4)$, gray zone lymphoma $(n=1)$, mediastinal lymphoma $(n=1)$ and high-grade lymphoma $(n=1)$.

Patients' demographic and clinical characteristics are shown in table 1 . As expected more pediatric patients were in the ALL population, resulting in a significantly lower age $(17 \pm 14$ years $)$ and weight $(44 \pm 21 \mathrm{~kg})$ compared with NHL patients $(44 \pm 15$ years and $75 \pm 20 \mathrm{~kg}$; p values $\leq 0.001)$. Both, ALL and NHL patients received an average of three prior lines of therapy. Thirty-two of 90 patients $(36 \%)$ received a hematopoietic stem cell transplantation prior CAR-T therapy, including 17 allogenic or haloidentical stem cell transplantations in patients with ALL 
Table 1 Baseline characteristics of treated patients

\begin{tabular}{lllll} 
& $\begin{array}{l}\text { Treated patients } \\
(\mathbf{n = 9 0 )}\end{array}$ & $\begin{array}{l}\text { ALL } \\
(\mathbf{n}=\mathbf{3 7})\end{array}$ & $\begin{array}{l}\text { NHL } \\
(\mathbf{n}=\mathbf{5 3})\end{array}$ & $\begin{array}{l}\text { P value } \\
\text { ALL vs NHL }\end{array}$ \\
\hline Mean age, years & $33 \pm 19$ & $17 \pm 14$ & $44 \pm 15$ & $\leq 0.001$ \\
Weight, $\mathrm{kg}$ & $62 \pm 25$ & $44 \pm 21$ & $75 \pm 20$ & $\leq 0.001$ \\
Gender - male, $\mathrm{n}(\%)$ & $61(68 \%)$ & $25(68 \%)$ & $36(68 \%)$ & 0.923 \\
CD3 blood count per $\mathrm{mm}^{3}$ & $1072 \pm 762$ & $1055 \pm 642$ & $1083 \pm 834$ & 0.871 \\
\hline
\end{tabular}

ALL, acute lymphoblastic leukemia; NHL, non-Hodgkin's lymphoma.

$(\mathrm{n}=15)$ and NHL $(\mathrm{n}=2)$. Ten of $37(27 \%)$ ALL patients received prior therapy directed against $\mathrm{CD} 19$, such as blinatumomab. ALL patients had significantly more CNS involvement $(\mathrm{p} \leq 0.001)$.

Following lympho-depleting preconditioning, fresh, non-cryopreserved CAR-T cells were intravenously infused to the patients. Clinical response was evaluated 1 to 2 months after CAR-T cell administration. One ALL patient died of sepsis before evaluation and one NHL patient is still awaiting his evaluation. Of 36 evaluated ALL patients, $24(67 \%)$ achieved minimal residual disease (MRD) negative CR, 6 (17\%) MRD positive CR and 5 patients (14\%) progressed. One ALL patient with an initial response was treated a second time with CAR-T, but did not respond. Of 52 evaluated NHL patients, $32(62 \%)$ achieved an objective response, including 16 complete remissions and 16 partial responses. Twenty (38\%) patients had disease progression.

\section{Leukapheresis, stimulation and expansion}

PBMC were collected by leukapheresis. The average CD3 T cell blood count before leukapheresis was 1,712 \pm 762 CD3 cells/ $\mu$ l, with no statistical difference between ALL and NHL patients $(\mathrm{p}=0.871)$ (table 2). Three ALL patients and one NHL patient with CD3 count below 250 cells $/ \mu 1$ were permitted to enroll to the study after approval by the

Table 2 Leukapheresis, production and infusion characteristics

\begin{tabular}{|c|c|c|c|c|}
\hline & $\begin{array}{l}\text { Treatments* } \\
(\mathrm{n}=91)\end{array}$ & $\begin{array}{l}\mathrm{ALL}^{*} \\
(\mathrm{n}=38)\end{array}$ & $\begin{array}{l}\text { NHL } \\
(n=53)\end{array}$ & $\begin{array}{l}\text { P value } \\
\text { ALL vs NHL }\end{array}$ \\
\hline \multicolumn{5}{|l|}{ Leukapheresis } \\
\hline CD3 + T cells, $(\%)$ & $53.3 \pm 24.5$ & $52 \pm 25.2$ & $54.3 \pm 24$ & 0.661 \\
\hline CD19 + B cells, $(\%)$ & $4.6 \pm 8.8$ & $7.8 \pm 10.9$ & $2.3 \pm 5.9$ & 0.003 \\
\hline \multicolumn{5}{|l|}{ Production } \\
\hline Cell number at initiation, $(x 10 \mathrm{e} 6)$ & $402 \pm 47$ & $405 \pm 72$ & $400 \pm 0$ & 0.620 \\
\hline Cell number on day 2, (x10e6) & $273 \pm 193$ & $304 \pm 171$ & $251 \pm 205$ & 0.203 \\
\hline Cell number for transduction, (x10e6) & $59.0 \pm 10.3$ & $58.2 \pm 9.5$ & $59.5 \pm 10.8$ & 0.555 \\
\hline Total cell number day 6, (x10e6) & $390 \pm 289$ & $475 \pm 313$ & $328 \pm 254$ & 0.017 \\
\hline Fold expansion day $6 \dagger$ & $6.9 \pm 5.7$ & $8.6 \pm 6.3$ & $5.7 \pm 4.9$ & 0.016 \\
\hline CAR-T day $6,(\%)$ & $58.7 \pm 17.4$ & $64.1 \pm 15.5$ & $54.8 \pm 17.6$ & 0.012 \\
\hline CAR-T cell number day $6,(x 10 \mathrm{e} 6)$ & $248 \pm 216$ & $319 \pm 230$ & $196 \pm 189$ & 0.007 \\
\hline CD19 + B cells day 6, (\%) & $0 \pm 0.2$ & $0 \pm 0.2$ & $0 \pm 0.3$ & 0.831 \\
\hline Total cell number day 10, (x10e6) & $1246 \pm 949$ & $1543 \pm 1104$ & $1033 \pm 750$ & 0.011 \\
\hline Fold expansion day $10 \dagger$ & $22.4 \pm 19.7$ & $28.7 \pm 24.7$ & $17.8 \pm 13.5$ & 0.009 \\
\hline CAR-T cell number day $10,(x 10 e 6)$ & $891 \pm 795$ & $1,174 \pm 901$ & $688 \pm 635$ & 0.004 \\
\hline \multicolumn{5}{|l|}{ Infusion product } \\
\hline CAR-T cells, (\%) & $67.1 \pm 19.1$ & $73.5 \pm 16.3$ & $62.6 \pm 19.6$ & 0.007 \\
\hline CD4 + CAR-T, (\%) & $29 \pm 18.1$ & $33 \pm 19.2$ & $26.1 \pm 16.6$ & 0.070 \\
\hline CD8 + CAR-T, (\%) & $71 \pm 18.1$ & $67 \pm 19.2$ & $73.9 \pm 16.6$ & 0.071 \\
\hline CD19 + B cells, (\%) & $0 \pm 0$ & $0 \pm 0$ & $0 \pm 0$ & 1 \\
\hline
\end{tabular}

${ }^{*}$ One ALL patient was treated twice.

†Fold expansion in comparison to the number of cells taken for transduction on day 2.

ALL, acute lymphoblastic leukemia; NHL, non-Hodgkin's lymphoma. 
A

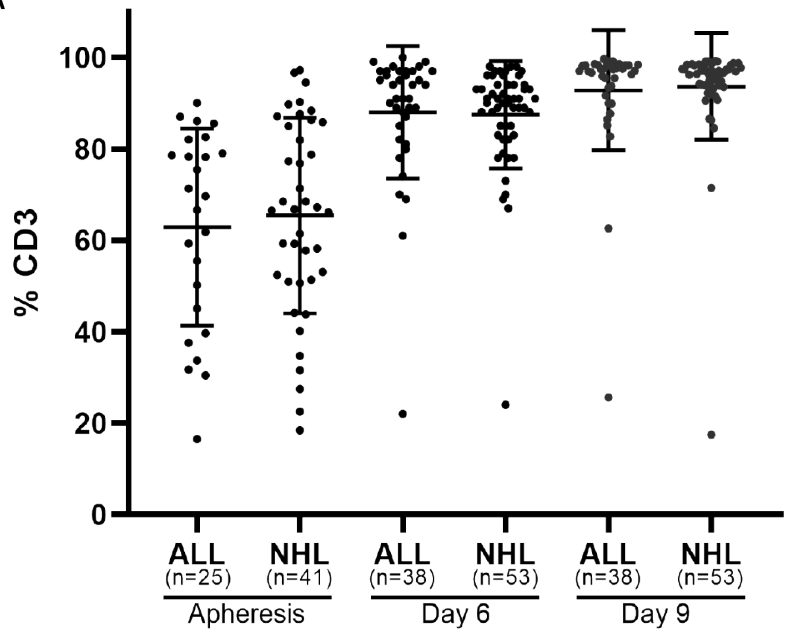

B

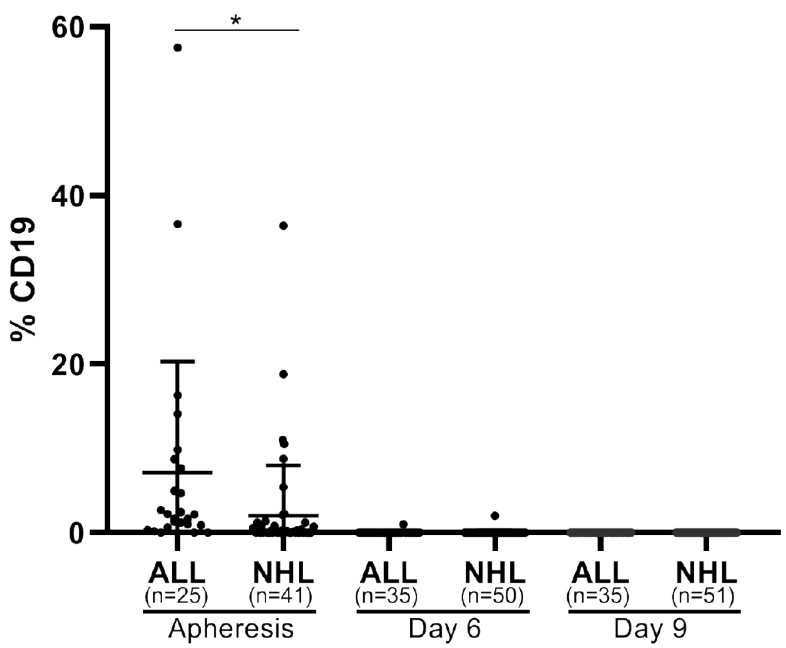

Figure 1 T cell and B-cell content during production. Frequency of CD3 T cells (A) and CD19 B cells $(B)$ at initiation (apheresis), during cell expansion (day 6), and before infusion (day 9) in ALL and NHL patients. ${ }^{*} \mathrm{p} \leq 0.05$.

Sheba Medical Center IRB due to rapid disease progression with no alternative treatment options. Ninety-one full manufacture processes were performed, including 38 productions for ALL patients (37 patients and 1 patient treated twice) and 53 NHL patients.

Time of apheresis procedure varied between 2 to 5 hours, depending on the weight and CD3 T cell blood count of the patient. The average number of mononuclear cells obtained by leukapheresis was $3.8 \pm 2.9 \times 10 \mathrm{e} 9$ (ALL patients, $2.7 \times 10^{9} \pm 2.7 \times 10^{9}$; NHL patients $4.5 \pm 3.0 \times 10^{9}$; $\mathrm{p} \leq 0.002)$. PBMC were purified by ficoll-hypaque density gradient and CD3 T cell and CD19 B-cell contents were determined by FACS analysis. The frequency of CD3 T cell in ALL and NHL patients was $62.9 \% \pm 21.1 \%$ (range $16 \%$ to $90 \%, \mathrm{n}=25$ ) and $65.2 \% \pm 21.1 \%$ (range $18 \%$ to $97 \%, \mathrm{n}=41)$, respectively, $(\mathrm{p}=0.640)$ and of CD19 B-cells $7.2 \% \pm 12.9 \%$ and $1.6 \% \pm 3.8 \%$ respectively $(\mathrm{p}=0.013)$ (figure 1, 'apheresis'). There was a strong correlation $(r=0.600)$ between the CD3 blood count and the CD3 frequency after ficol purification in the ALL population and a moderate correlation in the NHL population $(\mathrm{r}=0.572)$. Additional phenotype analysis on four apheresis products demonstrated the presence of monocytes $(26.4 \% \pm 14.0 \%)$ and NK cells $(13.4 \% \pm 6.0 \%)$.

CAR-T production was typically initiated with $400 \times 10 \mathrm{e} 6$ PBMC, obtained from fresh $(\mathrm{n}=84)$ or cryopreserved $(n=7)$ apheresis products. Only four collections of pediatric ALL patients under age 9 yielded less than $400 \times 10 \mathrm{e} 6$ PBMC and were initiated with less cells $(322 \times 10 \mathrm{e} 6$ on average) and one production was initiated with $600 \times 10 \mathrm{e} 6$ PBMC. PBMC were stimulated by addition of soluble anti CD3 antibody and IL-2. Since this condition stimulates CD3 T cells, but less other cell types, a drop in total cell number was typically observed after 2 days.

The cell count 2 days after stimulation dropped by approximately $30 \%$ and was $304 \pm 171 \times 10 \mathrm{e} 6$ in ALL patients and $251 \pm 205 \times 10 \mathrm{e} 6$ in NHL patients $(\mathrm{p}=0.203)$ (figure 2A, table 2). There was no correlation between the drop in cell number and the frequency of CD3 cells in the purified apheresis product $(\mathrm{R}=0.292)$. Transduction was typically performed with $60 \times 10 \mathrm{e} 6$ cells (range 48 to $72 \times 10 \mathrm{e} 6$ cells) on retronectin coated plates overnight and the remaining cells were discarded. On day 3 , cells were transferred to T175 or GRex100 flasks for expansion. Cell counts were performed on days 3, 6 and on day 10, the day of infusion. Figure 2A demonstrates the total cell number throughout the production and figure $2 \mathrm{~B}$ the fold expansion compared with day 2 .

As shown in figure 2A and B, cells of ALL patients expanded significantly better than cells of NHL patients. The fold expansion on day 6 compared with day 2 was 8.6 \pm 6.3 in ALL patients and $5.7 \pm 4.9$ in NHL patients

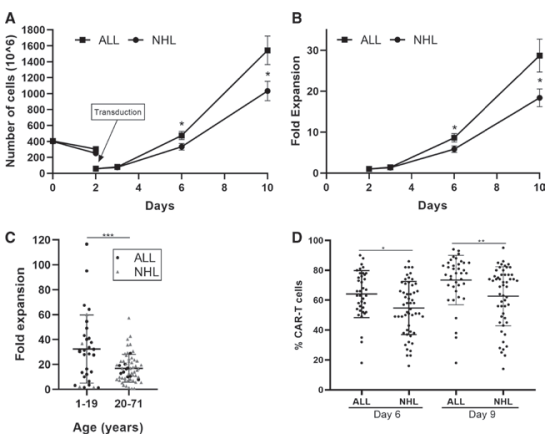

Figure 2 Comparison of the expansion capacity and transduction efficacy of CAR-T cells from ALL $(n=38)$ and $\mathrm{NHL}(\mathrm{n}=53)$ patients. (A) Total viable cell numbers $(x 10 \mathrm{e} 6)$ \pm SE. On day 2 only part of the cells were transduced and further expanded. (B) Fold expansion \pm SE in comparison to the number of cells taken for transduction on day 2. (C) Fold expansion of total viable cells according to age. (D) Transduction efficacy displayed as percent of CD3+F(ab) ${ }_{2}+$ CAR-T cells on day 6 and day $9 .{ }^{*} p \leq 0.01 ;{ }^{* *} p \leq 0.001$. ALL, acute lymphoblastic leukemia;CAR-T, chimeric antigen receptor T cells; NHL, non-Hodgkin's lymphoma. 
$(\mathrm{p}=0.016)$ and on day $10,28.7 \pm 24.7$ in ALL patients and $17.8 \pm 13.5$ in NHL patients $(p=0.009)$. Independent of the disease type, CAR-T cells of younger patients expanded significantly better, than of patients above age 21 (age $\leq 20,32.4 \pm 27.5$; age $>20,17.0 \pm 11.2 ; \mathrm{p}=0.0003$ ) (figure 2C).

On day 6 , the frequency of CD3 T cells was $87 \%$ in both patient populations and increased to $93 \% \pm 12 \%$ on day 10 (figure 1A). CD3 negative cells were mostly NK cells. CD19 + B-cell frequency was below $0.5 \%$ on day 6 and $0 \%$ on day 10 (figure 1B). Interestingly, transduction efficacy, measured by $\mathrm{CD} 3+\mathrm{F}(\mathrm{ab})_{2}+$ expression, was significantly higher in ALL patients than in NHL patients on day 6 (ALL, $64.1 \% \pm 15.5 \%$; NHL $54.8 \% \pm 17.6 \%$; $\mathrm{p}=0.012$ ) and on day $9 / 10$ (ALL, $73.5 \% \pm 16.3 \%$; NHL, $62.6 \% \pm 19.6 \%$, $\mathrm{p}=0.007$ ) (table 2, figure 2D).

Quality control testing required a minimum of $10 \%$ of CAR-T cells on day 6 and of $15 \%$ on day 9 or 10 . All 91 CAR-T products passed these criteria.

As a consequence of the significantly higher fold expansion and transduction efficacy in ALL patients, the total number of CAR-T cells on day 10 was almost double in ALL patients and reached $1,174 \pm 901 \times 10 \mathrm{e} 6$ cells compared with $688 \pm 635 \times 10 \mathrm{e} 6$ CAR-T cells in NHL patients $(\mathrm{p}=0.004)$.

The target dose of CAR-T cells for infusion in this study was $1 \times 10 \mathrm{e} 6$ CAR-T cells per $\mathrm{kg}$ body weight. Taking into account an average body weight of $44 \pm 21 \mathrm{~kg}$ and an average total number of $1,174 \pm 901 \times 10 \mathrm{e} 6 \mathrm{CAR}-\mathrm{T}$ cells in ALL patients, the in-house production yielded an average of 27-times more CAR-T cells than required. For NHL patients, an average of 9-times more CAR-T cells were obtained $(688 \pm 635 \times 10 \mathrm{e} 6 \mathrm{CAR}-\mathrm{T}$ cells and $75 \mathrm{~kg}$ $\pm 20 \mathrm{~kg}$ weight). The target dose of $1 \times 10 \mathrm{e} 6$ CAR-T cells was reached in $97 \%$ ( 88 of 91 ) of patients. In three NHL patients the CAR-T cell number on day 10 was $0.3 \times 10 \mathrm{e} 6$, $0.6 \times 10 \mathrm{e} 6$ and $0.8 \times 10 \mathrm{e} 6$ CAR-T per $\mathrm{kg}$. Following the approval by the Sheba Medical Center IRB, also these three patients received CAR-T therapy.

All 91 CAR-T products passed the required microbiological tests for sterility, endotoxin and mycoplasma, and no replication competent retrovirus was detected. The viability in all infusion products was above $90 \%$.

\section{Impact of age and gender}

As described before, CAR-T cells of patients below age 20 demonstrated a significantly increased fold expansion compared with older patients $(\mathrm{p}=0.0003$, figure $2 \mathrm{D})$. To further investigate the impact of age on production characteristics, ALL patients were divided into two age groups; age 1 to 19 years $(10.4 \pm 5.1$ years; $\mathrm{n}=28)$ and age 20 to 59 years ( $35.8 \pm 13.31$ years; $n=10)$. This analysis was not relevant for NHL patients, as only 3 of 53 patients were below the age of 20 years. As expected, the average number of mononuclear cells collected by leukapheresis $(p=0.029)$ and the weight $(p \leq 0.00001)$ was significantly lower in younger ALL patients. The fold expansion by day 10 was $33.4 \pm 27.6$ in ALL patients below age 20, and $15.7 \pm 6.17$ above age $20(\mathrm{p}=0.053)$. Young ALL patients had a significantly higher number of CAR-T cells on the day of infusion (patient age $\leq 19$ years, $1357 \pm 986 \times 10 \mathrm{e} 6$ cells; patient age $\geq 20$ years, $664 \pm 345 \times 10 \mathrm{e} 6$ cells; $\mathrm{p}=0.037$ ). The age had no impact on \% CD3 T cells frequency in the ficol-purified apheresis product $(\mathrm{p}=0.634)$, the transduction efficacy $(\mathrm{p}=0.625)$ or the $\mathrm{CD} 4 / \mathrm{CD} 8$ content in the infusion product $(\mathrm{p}=0.960)$.

Fifteen of 38 patients with ALL had a prior allogeneic or haloidentical hematopoietic stem cell transplant. When accounting for donor age in these patients, representing the biological age of the lymphocytes, results were similar, with significantly increased fold expansion (donor age $\leq 19$ years, 35.3 \pm 27.7 ; donor age $\geq 20$ years, $14.5 \pm 6.6$; $\mathrm{p}=0.015$ ) and a significantly higher number of CAR-T cells by day 10 (donor age $\leq 19$ years, $1436 \pm 979 \times 10 \mathrm{e} 6$ cells; donor age $\geq 20$ years, $609 \pm 345 \times 10 \mathrm{e} 6$ cells; $\mathrm{p}=0.008$ ) in younger patients. Of note, only two patients below age 19 received an allogeneic transplant from patients above age 20 .

Gender had no impact on any of the parameters.

ANCOVA analysis adjusted by age was applied to all ALL and NHL patients and confirmed that differences between CAR-T frequency in the infusion product were disease dependent (ALL vs NHL, $\mathrm{p}=0.031$ ), whereas cell count or fold expansion at day 10 were not disease dependent $(p=0.420)$, but rather effected by the age.

\section{Anti-tumor reactivity}

The potency of the CAR-T cells was evaluated after co-culture with CD19 expressing tumor cell lines (NALM-6, Toledo) followed by IFN $\gamma$ ELISA. In all cases, the IFN $\gamma$ levels after co-incubation with CD19 positive target cells were far above the required limit of $200 \mathrm{pg} / \mathrm{mL}$ IFN $\gamma$. Following co-culture with CD19 positive NALM-6 and Toledo, cells from ALL patients secreted an average of $4.5 \pm 4.8 \mathrm{mg} / \mathrm{mL}$ and $6.3 \pm 7.9 \mathrm{mg} / \mathrm{mL}$ IFN $\gamma$, respectively; and from NHL patients $8.5 \pm 12.7 \mathrm{mg} / \mathrm{mL}$ and $10.5 \pm 12.5 \mathrm{mg}$ $/ \mathrm{mL}$ IFN $\gamma$ respectively ( $\mathrm{p}$ values $>0.084$ ). There was no significant difference between the two patient populations. Untransduced T cells, or transduced T cells co-incubated with CD19 negative target line CCRF-CEM, resulted in an approximately 1000-times lower secretion. The results of this assay demonstrate the highly specific anti-tumor reactivity and potency of CAR-T cells.

\section{Phenotype analysis of CAR-T cells}

The differentiation status of CAR-T cells and expression of co-inhibitory molecules have previously been related to clinical response. ${ }^{26-29}$ Therefore, we analyzed headto-head the phenotype of ALL and NHL-derived CAR-T cells infusion products. CD8/CD4 subpopulation analysis of 91 infusion products did not reveal any significant differences between the two patient populations (CD8: ALL $67 \% \pm 19 \%$, NHL $74 \% \pm 17 \%, \mathrm{p}=0.071$; CD4: ALL $33 \% \pm 19 \%$, NHL $26 \% \pm 17 \%, \mathrm{p}=0.070$ ). Additional phenotype analysis was performed on 24 infusion samples of 12 ALL patients (seven MRD negative complete responders and five non-responders) and 12 NHL patients (six objective responders and six non-responders). The cells were 
analyzed for their differentiation status, determined by CCR7/CD45RA co-expression, expression of co-inhibitory receptors (PD-1, TIM-3, LAG-3), the co-stimulatory receptor CD28, the chemokine receptors CCR2, CCR4, CCR5, CXCR2, CXCR3 and the presence of gamma-delta $\mathrm{T}$ cells. Table 3 demonstrates the results gated on CAR-T cell of ALL versus NHL patients and online supplementary table 1 of clinical responders versus non-responders.

As shown in table 3 and figure $3 \mathrm{~A}$, infusion products from ALL patients contained significantly more CD45RA+CCR7+naïve (TN) CAR-T cells (ALL 60.5\% $\pm 17.3 \%$, $n=12$; NHL $41.2 \% \pm 22.4 \%, \mathrm{n}=12 ; \mathrm{p}=0.027)$ and significantly less CD45RA-CCR7+ central memory (TCM) CAR-T cells (ALL $15.8 \% \pm 11.8 \%, \mathrm{n}=12$; NHL $27.2 \% \pm 14.8 \%, \mathrm{n}=12$; $\mathrm{p}=0.048$ ). The co-inhibitory molecules PD-1, LAG-3 and TIM-3 and the co-stimulatory molecule CD28 were equally expressed on ALL and NHL-derived CAR-T cells. The average expression of TIM-3 was with $63.3 \% \pm 13.7 \%$ $(\mathrm{n}=24)$ almost double than of PD-1 $(32.4 \% \pm 11.6 \%, \mathrm{n}=24)$ and LAG-3 $(31.0 \% \pm 12.1 \%, \mathrm{n}=24)$. Also chemokine receptors were mostly equally expressed on CAR-T cells of ALL and NHL CAR-T, with the exception of CXCR3, which was significantly higher expressed in ALL patients (ALL $87.4 \% \pm 8.2 \%, \mathrm{n}=12$; NHL $79.1 \% \pm 11.1 \%, \mathrm{n}=12 ; \mathrm{p}=0.047)$. In general, the expression level of CXCR3 $(83.2 \% \pm 9.6 \%$, $\mathrm{n}=24$ ) was found to be the highest compared with other tested chemokine receptors (figure $3 \mathrm{~B}$ ).

We did not find any marker which was significantly different between responders and non-responders, besides the significantly higher expression of TIM-3 on CD8+ CAR-T cells of responding ALL patients (responders $38.8 \% \pm 12.4 \%, \mathrm{n}=7$; non-responders $25.2 \% \pm 5.4 \%, \mathrm{n}=5$; $\mathrm{p}=0.046)$.

\section{CAR-T infusion products compared to TIL infusion products}

Another type of adoptive $T$ cell therapy is the administration of tumor infiltrating lymphocytes. In comparison to CAR-T cells, TIL are not genetically-engineered and occur in lesions of solid tumor. TIL have a natural capability to recognize intracellular tumor antigens, which are presented as peptides on MHC molecules of the tumor cell. These tumor peptides may be shared between patients or derive from unique tumor mutations. ${ }^{30-32}$ Adoptive TIL therapy has mostly been described for patients with $\mathrm{r} / \mathrm{r}$ metastatic melanoma and yields objective response rates of $30 \%$ to $50 \%$ in a highly advanced patient population. ${ }^{20}{ }^{33-36}$ TIL T cells are isolated from tumor lesions, grown for about 1 month to generate a pure lymphocyte culture and further expanded in a rapid expansion procedure (REP). ${ }^{21}{ }^{24}$ REP uses similar reagents as the expansion of CAR-T cells; however, the addition of irradiated donor feeder cells at REP initiation induces an average fold expansion of about 1000-fold, compared with approximately 20-fold for CAR-T cells. Over the last 15 years we have manufactured over 100 TIL infusion products for melanoma patients. Although the production process of TIL and CAR-T cells largely differs, CAR-T and TIL are the two most common types of T cell
Table 3 Phenotype analysis of CD19 CAR-T cells infusion products of ALL and NHL patients

\begin{tabular}{|c|c|c|c|}
\hline & ALL $(n=12)$ & NHL $(n=12)$ & $P$ value \\
\hline \multicolumn{4}{|c|}{ T cell subpopulations } \\
\hline CD8+ & $59 \pm 15.6$ & $55.6 \pm 20.3$ & 0.652 \\
\hline CD4+ & $41 \pm 15.5$ & $44.4 \pm 20.3$ & 0.649 \\
\hline$\gamma \delta$ T cells & $1.0 \pm 0.8$ & $0.6 \pm 1.1$ & 0.140 \\
\hline \multicolumn{4}{|c|}{ Differentiation status } \\
\hline TN & $60.5 \pm 17.3$ & $41.2 \pm 22.4$ & 0.027 \\
\hline TCM & $15.8 \pm 11.8$ & $27.2 \pm 14.8$ & 0.048 \\
\hline TEM & $12.3 \pm 12.1$ & $22.4 \pm 13.8$ & 0.072 \\
\hline TEMRA & $11.4 \pm 5.8$ & $9.2 \pm 6.4$ & 0.399 \\
\hline
\end{tabular}

Co-stimulatory / co-inhibitory molecules

$\begin{array}{lccc}\text { CD28 + CD3+ } & 49.5 \pm 26.7 & 44.9 \pm 24.5 & 0.663 \\ \text { CD28 + CD8+ } & 37 \pm 19.5 & 29 \pm 14.5 & 0.267 \\ \text { CD28 + CD4+ } & 12.5 \pm 16 & 15.9 \pm 16.7 & 0.619 \\ \text { PD1 + CD3+ } & 30.3 \pm 13.4 & 34.5 \pm 9.7 & 0.382 \\ \text { PD1 + CD8+ } & 8.8 \pm 3.9 & 10.9 \pm 5.3 & 0.270 \\ \text { PD1 + CD4+ } & 21.5 \pm 12.3 & 23.6 \pm 10.2 & 0.652 \\ \text { LAG3 + CD3+ } & 26.5 \pm 11.3 & 35.5 \pm 13 & 0.082 \\ \text { LAG3 + CD8+ } & 19.8 \pm 11 & 25.9 \pm 13.4 & 0.243 \\ \text { LAG3 + CD4+ } & 6.6 \pm 2.5 & 9.7 \pm 5.5 & 0.097 \\ \text { TIM3 + CD3+ } & 65.8 \pm 14.8 & 60.9 \pm 12.7 & 0.394 \\ \text { TIM3 + CD8+ } & 33.1 \pm 11.9 & 28.8 \pm 8.9 & 0.327 \\ \text { TIM3 + CD4+ } & 32.7 \pm 14.4 & 32.1 \pm 15.3 & 0.925\end{array}$

\begin{tabular}{lccc}
\multicolumn{4}{l}{ Chemokine receptors } \\
CCR2 + CD3+ & $50.7 \pm 14.9$ & $50.5 \pm 15.9$ & 0.982 \\
\hline CCR2 + CD8+ & $31.7 \pm 14.1$ & $32.2 \pm 22.7$ & 0.957 \\
\hline CCR2 + CD4+ & $18.9 \pm 12.7$ & $18.4 \pm 12.7$ & 0.915 \\
\hline CCR5 + CD3+ & $10.5 \pm 8.8$ & $16 \pm 15.9$ & 0.303 \\
CCR5 + CD8+ & $2.9 \pm 3.7$ & $6.1 \pm 8.2$ & 0.233 \\
\hline CCR5 + CD4+ & $7.6 \pm 5.4$ & $10 \pm 9.5$ & 0.464 \\
CCR4 + CD3+ & $33.6 \pm 17.2$ & $36.1 \pm 11.5$ & 0.682 \\
CCR4 + CD8+ & $14.5 \pm 10.1$ & $15.9 \pm 12.7$ & 0.763 \\
\hline CCR4 + CD4+ & $19.1 \pm 13.4$ & $20.1 \pm 11.4$ & 0.838 \\
CXCR2 + CD3+ & $17.7 \pm 9.8$ & $15.5 \pm 5.4$ & 0.499 \\
CXCR2 + CD8+ & $4.6 \pm 7.5$ & $3.8 \pm 3.5$ & 0.743 \\
CXCR2 + CD4+ & $13.1 \pm 8.2$ & $11.6 \pm 5.2$ & 0.618 \\
CXCR3 + CD3+ & $87.4 \pm 8.2$ & $79.1 \pm 11.1$ & 0.047 \\
CXCR3 + CD8+ & $52.6 \pm 14.9$ & $48.3 \pm 21.6$ & 0.580 \\
CXCR3 + CD4+ & $34.9 \pm 13.4$ & $30.8 \pm 12.6$ & 0.448 \\
\hline
\end{tabular}

Cells were gated on $\mathrm{CD} 3+\mathrm{F}(\mathrm{ab})_{2}+\mathrm{CAR}-\mathrm{T}$ cells. TN (naïve $T$ cells), CD3+CD45RA+CCR7+; TCM (central memory $T$ cells), CD3+CD45RA-CCR7+; TEM (effector memory T cells), CD3+CD45RA-CCR7-; TEMRA (T cells effector), CD3+CD45RA+CCR7-.

ALL, acute lymphoblastic leukemia; CAR-T, chimeric antigen receptor T cells; NHL, non-Hodgkin's lymphoma. 


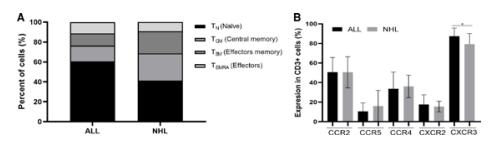

Figure 3 Phenotype analysis of CAR-T infusion products derived from ALL $(n=12)$ and NHL $(n=12)$ patients. Cells were gated on $\mathrm{CD} 3+\mathrm{F}(\mathrm{ab})_{2}+\mathrm{CAR}-\mathrm{T}$ cells. (A) Differentiation status. TN (naïve T cells), CD3+CD45RA+CCR7+; TCM (central memory T cells), CD3+CD45RA-CCR7+; TEM (effector memory T cells), CD3+CD45RA-CCR7-; TEMRA (effector T cells), CD3+CD45RA+CCR7-. (B) Chemokine receptor profile. ${ }^{*} p<0.01$. SD are presented. ALL, acute lymphoblastic leukemia; CAR-T,chimeric antigen receptor T cells; NHL, nonHodgkin's lymphoma.

therapy to date. We therefore compared the phenotype of CAR-T infusion products $(\mathrm{n}=24)$ with TIL infusion products $(\mathrm{n}=40-88)$. As shown in table 4 , TIL and CAR products had a similar content of CD4 and CD8 T cells, as well as PD1 and CD28 expressing T cells. CAR-T cells demonstrated a significantly higher expression of LAG-3 (CAR-T, $25 \% \pm 11 \%$; TIL, $10 \% \pm 4 \%$; $\mathrm{p} \leq 0.001$ ) and significantly lower expression of TIM-3 (CAR-T $50 \% \pm 15 \%$; TIL, $61 \% \pm 14 \% ; \mathrm{p}=0.004)$. As expected, TIL demonstrate a more differentiated phenotype and were almost exclusively (97\%) effector memory T cells, whereas $49 \% \pm 18 \%$ and $17 \% \pm 14 \%$ of CAR-T cells were naive or central

Table 4 Comparison of CAR-T and TIL infusion products

\begin{tabular}{|c|c|c|c|}
\hline & $\begin{array}{l}\text { CAR-T } \\
n=24\end{array}$ & TIL $(n=40-88)$ & $P$ value \\
\hline \multicolumn{4}{|c|}{ T cell subpopulations } \\
\hline CD8+ & $58 \pm 16$ & $63 \pm 24(n=88)$ & 0.386 \\
\hline CD4+ & $42 \pm 17$ & $35 \pm 24(n=88)$ & 0.200 \\
\hline \multicolumn{4}{|c|}{ Differentiation status } \\
\hline TN & $49 \pm 18$ & $0.05 \pm 0.10(n=88)$ & $\leq 0.001$ \\
\hline TCM & $17 \pm 14$ & $2.3 \pm 2.5(n=88)$ & $\leq 0.001$ \\
\hline TEM & $18 \pm 13$ & $96.8 \pm 3.0(n=88)$ & $\leq 0.001$ \\
\hline TEMRA & $15 \pm 13$ & $0.82 \pm 1.5(n=88)$ & $\leq 0.001$ \\
\hline \multicolumn{4}{|c|}{ Co-stimulatory / co-inhibitory molecules } \\
\hline CD28 + CD3+ & $47 \pm 26$ & $52 \pm 20(n=88)$ & 0.396 \\
\hline $\mathrm{PD} 1+\mathrm{CD} 3+$ & $26 \pm 8$ & $26 \pm 15(n=40)$ & 0.494 \\
\hline LAG3 + CD3+ & $25 \pm 11$ & $10 \pm 4(n=40)$ & $\leq 0.001$ \\
\hline TIM3 + CD3+ & $50 \pm 15$ & $61 \pm 14(n=40)$ & 0.004 \\
\hline \multicolumn{4}{|c|}{ Chemokine receptors } \\
\hline $\mathrm{CCR} 5+\mathrm{CD} 3+$ & $12 \pm 13$ & $80 \pm 24(n=56)$ & $\leq 0.001$ \\
\hline $\mathrm{CCR} 4+\mathrm{CD} 3+$ & $33 \pm 14$ & $27 \pm 12(n=88)$ & 0.032 \\
\hline CXCR3 + CD3+ & $79 \pm 12$ & $86 \pm 20(n=88)$ & 0.076 \\
\hline
\end{tabular}

Phenotype analysis of 24 CAR-T cells and 40 to 88 TIL infusion products. Cells were gated on viable cells. TIL, tumor infiltrating lymphocytes, TN (naïve T cells), CD3+CD45RA+CCR7+; TCM (central memory T cells), CD3+CD45RA-CCR7+; TEM (effector memory T cells), CD3+CD45RA-CCR7-; TEMRA (effector T cells), CD3+CD45RA+CCR7-.

CAR-T, chimeric antigen receptor $\mathrm{T}$ cells. memory $\mathrm{T}$ cells, respectively. The chemokine receptor CCR5 was significantly higher expressed on TIL (CAR-T, $12 \% \pm 13 \%$; TIL, $80 \% \pm 24 \%$; $\leq 0.001$ ).

\section{DISCUSSION}

CD19 CAR-T cells have demonstrated high effectiveness for the treatment of $\mathrm{r} / \mathrm{r}$ ALL and NHL patients, leading to FDA approval of two commercial products in 2017. Although highly potent, the commercial CD19 CAR-T products have the disadvantage of being very costly. In addition, as a consequence of the high turnaround time of 1 to 2 months from leukapheresis to infusion, many rapidly progressive patients clinically deteriorate during the waiting period. The JULIET and ELIANA phase 3 trials with tisagenlecleucel reported an intent-to-treat analysis from screening to treatment of only $47 \%$ and $70 \%$, respectively. ${ }^{57}$ As a result, a rising number of clinical centers produce their own CAR-T cells in-house. To date, the Sheba Medical Center, Israel, has conducted one of the highest numbers of treatments with in-house produced CD19 CAR-T cells and TIL worldwide. All patients were heavily pretreated and CAR-T cells were mostly given as salvage therapy. We demonstrated that the short turnaround time of only 9 to 10 days, enabled the treatment of almost all enrolled patients. Only 2 of 93 (2\%) patients dropped out due to clinical deterioration. The production success was $99 \%$ (91 of 92).

In this study, the CAR-T production platform was identical for ALL and NHL patients, allowing true comparison between cells from different patient populations. Although the average CD3 blood counts were very similar in ALL and NHL patients $(p=0.871)$ at the time of leukapheresis, CAR-T cells from ALL patients expanded significantly better than of NHL patients ( $17.8 \pm 13.5$ fold) and reached a fold expansion of $28.7 \pm 24.7$ compared with day $2(\mathrm{p}=0.009)$. This difference was explained by the variance of age rather than the disease type. The transduction efficacy was higher in ALL derived infusion products than in NHL ( $p=0.007)$, independent of age. The target CAR-T cell number in this trial was $1 \times 10 \mathrm{e} 6$ CAR-T cells per kilogram weight, since this dose was found effective and relatively safe in adults with $\mathrm{NHL}^{38}$ and children and young adults with ALL. ${ }^{39}$

As a consequence of the younger age, the lower weight, the better expansion rate and the higher transduction efficacy, an average of 27-times more CAR-T cells were produced for ALL patients than required. For NHL patients 9-times more CAR-T cells were manufactured on average. Three NHL patients received a dose below $1 \times 10 \mathrm{e} 6 / \mathrm{kg}$, compared with none of the ALL patients.

The produced CAR-T cells were highly potent and secreted IFN $\gamma$ in the range of milligrams per $\mathrm{ml}$ after co-culture with CD19 expressing tumor lines. Specificity was confirmed by at least a 1000 -times lower secretion of IFN $\gamma$, when co-incubated with a CD19 negative tumor line. 
The infused cell products were clinically effective, leading to remission in $84 \%$ of the ALL patients and $62 \%$ of evaluated NHL patients.

The differentiation status analysis of ALL derived CAR-T cells revealed a higher content of naïve $\mathrm{T}$ cells, whereas CAR-T cells of NHL patients had a higher frequency of central memory $\mathrm{T}$ cells. Both, naïve and central memory $\mathrm{T}$ cells were shown to enhance the potency and durability of CAR-T cells. ${ }^{2640}$ However, naïve CD4 T cells were reported to secrete more cytokines than TCM. ${ }^{26}$ Other than that, ALL and NHL-derived CAR-T had a very similar phenotype, except for the chemokine receptor CXCR3, which was significantly higher expressed on CAR-T cells derived from ALL patients. Due to alternative splicing of the CXCR3 mRNA, three variants have been reported, CXCR3-A, CXCR3-B, and CXCR3-Alt, which exhibit opposing cellular effects. ${ }^{41}$ To understand the potential implication of a higher expression level of CXCR3 in CAR-T products derived from ALL patients, further investigation will be required.

CAR-T therapy has emerged as a promising approach for hematological malignancies, but is yet less effective in solid tumors. Challenges which need to be overcome in solid tumors, include tumor target selection, the immunosuppressive tumor-microenvironment, tumor heterogeneity and inadequate intratumoral $\mathrm{T}$ cell trafficking. ${ }^{42-44}$ Adoptive T cell therapy with TIL on the other hand, has demonstrated high objective response rates in melanoma and other solid tumors. ${ }^{20}{ }^{33-36}$ Analyzing differences of TIL and CAR-T cell infusion products, might provide a way to gain insight and improve CAR-T cell therapies for solid tumors. Interestingly, FACS analysis of CAR-T and TIL infusion products revealed a more differentiated phenotype in TIL (97\% effector memory T cells) and higher expression of CCR5. This may indicate a lower persistence of TIL, but also a higher infiltration capacity. Further analysis will be required. Of note, it was shown that CCL3, CCL4, and CCL5, the corresponding ligands of CCR5, are a major determinant of immune cellular infiltration in tumors. ${ }^{45}$ Since active trafficking of $\mathrm{T}$ cells into tumor mass partially depends on the interaction between chemokines in tumor and chemokine receptors presented on T cells, new CAR-T cell therapies armed with chemokine receptors are currently under development. ${ }^{4647}$

\section{CONCLUSIONS}

The in-house production of CAR-T cells is highly efficient. CAR-T cells were successfully produced for $99 \%$ of patients in just 9 to 10 days, which allowed the treatment of $97 \%$ of enrolled patients. CAR-T cells of ALL patients demonstrated increased proliferation and transduction capacity compared with NHL-derived CAR-T cells, which may explain improved clinical remission in these patients.

AcknowledgmentThe authors would like to thank Mrs Rina Sareli and Mrs Bella Baturov from the leukapheresis units and Ran Avrahami for performing the ANCOVA analysis. The authors would also like to thank the Lemelbaum family for their generous support.

Contributors MJB, OI, and EJ designed the study. EJ, AT, AA, ANagler, GM, and JS recruited and treated the patients, and are investigators. OI, ANissani, ML, AK, KB, $H B, L Z, H V$, and MR collected and analyzed the data. OI, ANissani, ML, AK, KB, HB, LZ, and MJB led the production and quality control of the in-house CAR-T cell and TIL program. OI, ANissani, and MJB wrote the paper. All authors read and approved the final manuscript.

Funding The clinical trial was funded by the Sheba Medical Center and a donation of the Lemelbaum family.

Competing interests None declared.

Patient consent for publication Not required.

Ethics approval The study has been performed in accordance with the Declaration of Helsinki and was approved by the Israeli Ministry of Health Approval numbers: 20 161748 (CAR-T) and SMC356616 (TIL). An informed consent to participate in the study was obtained from all participants or their legal guardian for minors.

Provenance and peer review Not commissioned; externally peer reviewed. Data availability statement Data are available upon reasonable request.

Open access This is an open access article distributed in accordance with the Creative Commons Attribution Non Commercial (CC BY-NC 4.0) license, which permits others to distribute, remix, adapt, build upon this work non-commercially, and license their derivative works on different terms, provided the original work is properly cited, appropriate credit is given, any changes made indicated, and the use is non-commercial. See http://creativecommons.org/licenses/by-nc/4.0/.

ORCID iD

Michal J Besser http://orcid.org/0000-0002-9233-0458

\section{REFERENCES}

1 van Loenen MM, de Boer R, Amir AL, et al. Mixed T cell receptor dimers harbor potentially harmful neoreactivity. Proc Natl Acad Sci U S A 2010;107:10972-7.

2 Gross G, Waks T, Eshhar Z. Expression of immunoglobulin-T-cell receptor chimeric molecules as functional receptors with antibodytype specificity. Proc Natl Acad Sci U S A 1989;86:10024-8.

3 Kuwana Y, Asakura Y, Utsunomiya N, et al. Expression of chimeric receptor composed of immunoglobulin-derived $\mathrm{V}$ regions and T-cell receptor-derived C regions. Biochem Biophys Res Commun 1987;149:960-8.

4 Locke FL, Ghobadi A, Jacobson CA, et al. Long-Term safety and activity of axicabtagene ciloleucel in refractory large B-cell lymphoma (ZUMA-1): a single-arm, multicentre, phase 1-2 trial. Lancet Oncol 2019;20:31-42.

5 Maude SL, Laetsch TW, Buechner J, et al. Tisagenlecleucel in children and young adults with B-cell lymphoblastic leukemia. $N$ Engl $J$ Med 2018;378:439-48.

6 Maude SL, Frey N, Shaw PA, et al. Chimeric antigen receptor T cells for sustained remissions in leukemia. $N$ Engl $\mathrm{J}$ Med 2014;371:1507-17.

7 Schuster SJ, Svoboda J, Chong EA, et al. Chimeric antigen receptor T cells in refractory B-cell lymphomas. N Engl J Med 2017;377:2545-54.

8 Pui C-H, Yang JJ, Hunger SP, et al. Childhood acute lymphoblastic leukemia: progress through collaboration. J Clin Oncol 2015;33:2938-48.

9 Fielding AK, Richards SM, Chopra R, et al. Outcome of 609 adults after relapse of acute lymphoblastic leukemia (all); an MRC UKALL12/ECOG 2993 study. Blood 2007;109:944-50.

10 Ferlay J, Soerjomataram I, Dikshit R, et al. Cancer incidence and mortality worldwide: sources, methods and major patterns in GLOBOCAN 2012. Int J Cancer 2015;136:E359-86.

11 Chihara D, Ito $\mathrm{H}$, Matsuda $\mathrm{T}$, et al. Differences in incidence and trends of haematological malignancies in Japan and the United States. Br J Haematol 2014;164:536-45.

12 Flowers CR, Sinha R, Vose JM. Improving outcomes for patients with diffuse large B-cell lymphoma. CA Cancer J Clin 2010;60:393-408.

13 Abbott BL. Advances in the diagnosis and treatment of chronic lymphocytic leukemia. Clinical advances in hematology \& oncology: H\&O 2004;2:448-54.

14 Jacoby E, Bielorai B, Avigdor A, et al. Locally produced CD19 CAR $T$ cells leading to clinical remissions in medullary and extramedullary 
relapsed acute lymphoblastic leukemia. Am J Hematol 2018;93:1485-92.

15 Avigdor A, Shouval R, Jacoby E, et al. Car T cells induce a complete response in refractory Burkitt lymphoma. Bone Marrow Transplant 2018;53:1583-5

16 Feldman SA, Goff SL, Xu H, et al. Rapid Production of Clinical-Grade Gammaretroviral Vectors in Expanded Surface Roller Bottles Using a "Modified" Step-Filtration Process for Clearance of Packaging Cells. Hum Gene Ther 2011;22:107-15.

17 Kochenderfer JN, Feldman SA, Zhao Y, et al. Construction and preclinical evaluation of an anti-CD19 chimeric antigen receptor. Journal of immunotherapy 2009;32:689-702.

18 Nicholson IC, Lenton KA, Little DJ, et al. Construction and characterisation of a functional CD19 specific single chain Fv fragment for immunotherapy of B lineage leukaemia and lymphoma. Mol immunology 1997;34:1157-65.

19 Bajgain P, Mucharla R, Wilson J, et al. Optimizing the production of suspension cells using the G-Rex "M" series. Molecular Therapy Methods \& Clinical Development 2014;1:14015.

20 Besser MJ, Shapira-Frommer R, Itzhaki O, et al. Adoptive transfer of tumor-infiltrating lymphocytes in patients with metastatic melanoma: intent-to-treat analysis and efficacy after failure to prior immunotherapies. Clinical Cancer Research 2013;19:4792-800.

21 Itzhaki O, Hovav E, Ziporen Y, et al. Establishment and Largescale Expansion of Minimally cultured "Young" Tumor Infiltrating Lymphocytes for Adoptive Transfer Therapy. J Immunother 2011;34:212-20.

22 Tran KQ, Zhou J, Durflinger KH, et al. Minimally cultured tumorinfiltrating lymphocytes display optimal characteristics for adoptive cell therapy. Journal of immunotherapy 2008;31:742-51.

23 Dudley ME, Yang JC, Sherry R, et al. Adoptive cell therapy for patients with metastatic melanoma: evaluation of intensive myeloablative chemoradiation preparative regimens. JCO 2008;26:5233-9.

24 Besser MJ, Shapira-Frommer R, Treves AJ, et al. Clinical responses in a phase II study using adoptive transfer of short-term cultured tumor infiltration lymphocytes in metastatic melanoma patients. Clinical Cancer Res 2010;16:2646-55.

25 Besser MJ, Shapira-Frommer R, Treves AJ, et al. Minimally cultured or selected autologous tumor-infiltrating lymphocytes after a lymphodepleting chemotherapy regimen in metastatic melanoma patients. Journal of Immunotherapy 2009;32:415-23.

26 Sommermeyer D, Hudecek M, Kosasih PL, et al. Chimeric antigen receptor-modified $\mathrm{T}$ cells derived from defined CD8+ and CD4+ subsets confer superior antitumor reactivity in vivo. Leukemia 2016;30:492-500.

27 Xu Y, Zhang M, Ramos CA, et al. Closely related T-memory stem cells correlate with in vivo expansion of CAR.CD19-T cells and are preserved by IL-7 and IL-15. Blood 2014;123:3750-9.

28 Klaver Y, van Steenbergen SCL, Sleijfer S, et al. T cell maturation stage prior to and during GMP processing informs on CAR T cell expansion in patients. Front Immunol 2016;7:648.

29 Oliveira G, Ruggiero E, Stanghellini MTL, et al. Tracking genetically engineered lymphocytes long-term reveals the dynamics of $\mathrm{T}$ cell immunological memory. Sci Transl Med 2015;7:317ra198.

30 Samuels Y, Kalaora S, Wolf Y, et al. Combined analysis of antigen presentation and $T$ cell recognition reveals restricted immune responses in melanoma. Cancer Discovery 2018:CD-17-1418.
31 Simon S, Wu Z, Cruard J, et al. Tcr analyses of two vast and shared melanoma antigen-specific $T$ cell repertoires: common and specific features. Front Immunol 1962;2018:9.

32 Yossef R, Tran E, Deniger DC, et al. Enhanced detection of neoantigen-reactive $T$ cells targeting unique and shared oncogenes for personalized cancer immunotherapy. JCI Insight 2018;3.

33 Dudley ME, Gross CA, Somerville RPT, et al. Randomized Selection Design Trial Evaluating CD8 ${ }^{+}$-Enriched Versus Unselected TumorInfiltrating Lymphocytes for Adoptive Cell Therapy for Patients With Melanoma. JCO 2013;31:2152-9.

34 Forget MA, Haymaker C, Hess KR, et al. Prospective analysis of adoptive TIL therapy in patients with metastatic melanoma: response, impact of anti-CTLA4, and biomarkers to predict clinical outcome. Clin Cancer Res 2018;24:4416-28.

35 Goff SL, Dudley ME, Citrin DE, et al. Randomized, prospective evaluation comparing intensity of Lymphodepletion before adoptive transfer of tumor-infiltrating lymphocytes for patients with metastatic melanoma. JCO 2016;34:2389-97.

36 Rosenberg SA, Yang JC, Sherry RM, et al. Durable complete responses in heavily pretreated patients with metastatic melanoma using T-cell transfer immunotherapy. Clinical Cancer Res 2011;17:4550-7.

37 Schuster SJ, Bishop MR, Tam CS, et al. Tisagenlecleucel in adult relapsed or refractory diffuse large B-cell lymphoma. N Engl J Med 2019;380:45-56.

38 Kochenderfer JN, Dudley ME, Kassim SH, et al. Chemotherapyrefractory diffuse large B-cell lymphoma and indolent B-cell malignancies can be effectively treated with autologous T cells expressing an anti-CD19 chimeric antigen receptor. J Clin Oncol 2015;33:540-9.

39 Lee DW, Kochenderfer JN, Stetler-Stevenson M, et al. T cells expressing CD19 chimeric antigen receptors for acute lymphoblastic leukaemia in children and young adults: a phase 1 dose-escalation trial. Lancet 2015;385:517-28.

40 Finney OC, Brakke HM, Rawlings-Rhea S, et al. Cd19 CAR T cell product and disease attributes predict leukemia remission durability. J Clin Invest 2019;129:2123-32.

41 Reynders N, Abboud D, Baragli A, et al. The distinct roles of CXCR3 variants and their ligands in the tumor microenvironment. Cells 2019;8:613

42 Shiao SL, Ganesan AP, Rugo HS, et al. Immune microenvironments in solid tumors: new targets for therapy. Genes Dev 2011;25:2559-72.

43 Sackstein R, Schatton T, Barthel SR. T-Lymphocyte homing: an underappreciated yet critical hurdle for successful cancer immunotherapy. Laboratory Investigation 2017;97:669-97.

44 Mata M, Gottschalk S. Engineering for Success: Approaches to Improve Chimeric Antigen Receptor T Cell Therapy for Solid Tumors. Drugs 2019;79:401-15.

45 Allen F, Bobanga ID, Rauhe P, et al. CCL3 augments tumor rejection and enhances $\mathrm{CD} 8{ }^{+} \mathrm{T}$ cell infiltration through $\mathrm{NK}$ and $\mathrm{CD}_{103}{ }^{+}$dendritic cell recruitment via IFN $\gamma$. Oncoimmunology 2018;7:e1393598.

46 Craddock JA, Lu A, Bear A, et al. Enhanced tumor trafficking of GD2 chimeric antigen receptor T cells by expression of the chemokine receptor CCR2B. Journal of Immunotherapy 2010;33:780-8.

47 Jin L, Tao H, Karachi A, et al. CXCR1- or CXCR2-modified CAR T cells co-opt IL-8 for maximal antitumor efficacy in solid tumors. Nat Commun 2019;10:4016. 